\title{
Atherogenic lipoprotein phenotype and LDL size and subclasses in drug-naïve patients with early rheumatoid arthritis
}

\author{
Manfredi Rizzo ${ }^{\mathrm{a}, *, 1}$, Giatgen A Spinas ${ }^{\mathrm{b}}$, Mustafa Cesur ${ }^{\mathrm{c}}$, Zeynep Ozbalkan ${ }^{\mathrm{d}}$, \\ Giovam Battista Rini ${ }^{a}$, Kaspar Berneis ${ }^{\mathrm{b}, 1}$ \\ a Department of Internal Medicine and Emerging Diseases, University of Palermo, Palermo, Italy \\ b Division of Endocrinology, Diabetes \& Clinical Nutrition, University Hospital Zurich, Zurich, Switzerland \\ c Department of Endocrinology and Metabolic Diseases, Ankara Guven Hospital, Ankara, Turkey \\ d Department of Rheumatology, Ankara Numune Education and Research Hospital, Ankara, Turkey
}

\section{A R T I C L E I N F O}

\section{Article history:}

Received 9 February 2009

Received in revised form 30 June 2009

Accepted 1 July 2009

Available online 8 July 2009

\section{Keywords:}

Rheumatoid arthritis

small

dense LDL

HDL-cholesterol

Triglycerides

\begin{abstract}
A B S T R A C T
Objective: : Subjects with rheumatoid arthritis (RA) have increased cardiovascular risk and may show atherogenic forms of dyslipidemia. The present study investigated whether patients with early RA, beyond alterations in plasma lipids, also show lower LDL size and altered LDL subclass distribution. Design and Methods: We identified 25 subjects with RA ( $47 \pm 8$ years, body mass index (BMI) $25 \pm 4 \mathrm{~kg} / \mathrm{m}^{2}$ ) by the American College of Rheumatology diagnostic criteria, with a disease durations $<1$ year and no prior treatment against it. In patients and 22 healthy subjects matched for age and BMI (controls) we measured plasma lipids and LDL size and subclasses by gradient gel electrophoresis.

Results: As compared to controls RA patients had higher plasma triglycerides $(1.8 \pm 0.5 \mathrm{vs} .1 .0 \pm 0.5 \mathrm{mmol} / \mathrm{L}$ $p<0.0001)$ and lower HDL-cholesterol concentrations ( $1.2 \pm 0.2$ vs. $1.4 \pm 0.2 \mathrm{mmol} / \mathrm{L}, p=0.0027)$, while total- and LDL-cholesterol concentrations were similar. LDL particle size was lower in RA patients than controls ( $264 \pm 7$ vs. $281 \pm 9 \AA$, $p<0.0001$ ), due to less LDL-I ( $31 \pm 6$ vs. $38 \pm 7 \%, p=0.0004)$ and LDLIIA ( $14 \pm 3$ vs. $16 \pm 3 \%, p=0.0182$ ), and more LDL-IIIB ( $7 \pm 1$ vs. $5 \pm 1 \%$ ), -IVA ( $11 \pm 2$ vs. $8 \pm 2 \%$ ) and -IVB particles ( $12 \pm 2$ vs. $9 \pm 2 \%$, $(p<0.0001$ for all). Further, about $1 / 3$ of patients showed the complete "atherogenic-lipoprotein-phenotype" (e.g. the concomitant presence of high triglycerides, low HDLcholesterol and elevated small, dense LDL).

Conclusions: Beyond plasma lipids, increased levels of small, dense LDL seems to be common in drugnaïve patients with early RA. Yet, whether these findings affect the atherogenic process and the clinical endpoints in these subjects remains to be determined by future prospective studies.
\end{abstract}

(C) 2009 Elsevier Ireland Ltd. All rights reserved.

\section{Introduction}

Patients with rheumatoid arthritis (RA) have increased cardiovascular morbidity and mortality as compared to the general population [1-3]. Among the traditional cardiovascular risk factors, the lipid profile in RA has often been described as "proatherogenic" based on decreased HDL-cholesterol and increased LDL:HDLcholesterol ratio [4,5]. In recent years it has become evident that the lipid triad or "atherogenic lipoprotein phenotype" (ALP), characterized by decreased HDL-cholesterol, moderately raised triglycerides and increased levels of small, dense LDL, is

\footnotetext{
* Corresponding author at: Dipartimento di Medicina Clinica e delle Patologie Emergenti, Universita' di Palermo, Via del Vespro, 141 - 90127, Palermo, Italy. Tel.: +39091 6552945; fax: +390916552982.

E-mail address: mrizzo@unipa.it (M. Rizzo).

1 These two authors contributed equally to the present work.
}

linked to increased cardiovascular risk, beyond LDL-cholesterol levels [6-8]. In fact, a number of evidences suggest that the quality, and not only the quantity, of LDL exerts a direct influence on cardiovascular risk [9] and the predominance of small dense LDL has been accepted as an emerging cardiovascular risk factor by the National Cholesterol Education Program Adult Treatment Panel III [10].

It has been reported in a previous study that patients with long-term RA may show lower LDL size due to increased levels of small, dense LDL particles [11]. Yet, this has not been further investigated in patients with early RA, without prior treatment for it, which limits the utility of the information we have on atherogenic dyslipidemia in RA. In fact, although drug treatment in RA is in general beneficial on plasma lipids, it seems that long-term anti-tumor necrosis factor-alpha therapies may induce some proatherogenic changes in lipid profile [12-14]. Therefore, we included in the present study a group of patients with early RA, without treatment, as well as a control group of healthy subjects, matched for age and body mass index (BMI) used as controls, in order to 
investigate (1) whether patients with early RA have lower LDL size; (2) whether their LDL subclass distribution is altered (i.e. increased levels of small, dense LDL); (3) the prevalence of ALP.

\section{Methods}

\subsection{Subjects and study protocol}

We included in the present study a group of drug-naïve patients with early RA who consecutively underwent a clinical examination at the Department of Rheumatology, Ankara Numune Education and Research Hospital, Ankara, Turkey between January and June 2008. Inclusion criteria were the fulfillment of the American College of Rheumatology diagnostic criteria for RA [15] and a disease durations $<1$ year. Disease activity was assessed by measuring the 28 joint indices score (DAS-28) [16]. According to these inclusion criteria we have found 144 patients potentially eligible for our study. We then excluded subjects with prior use of any treatment for RA, the presence of abnormal liver, renal or thyroid function, type-2 diabetes, cardiovascular diseases, active neoplasia, chronic systemic or inflammatory diseases, or therapy with drugs known to affect lipid metabolism. According to these exclusion criteria we were able to include in our study 25 drug-naïve patients with early RA. As controls we included 22 healthy subjects with the same exclusion criteria and matched for age and BMI, in order to have a more consistent group with that of patients. Controls were coworkers or family co-workers of the Ankara Numune Education and Research Hospital, Turkey. The study was approved by the Ethics Committee of Ankara Numune Education and Research Hospital, Turkey and each subject gave written informed consent to participate.

\subsection{Biochemistry}

A blood sample was collected from each subject after $12-14 \mathrm{~h}$ overnight fast in sodium-EDTA tubes and stored at $-80^{\circ} \mathrm{C}$ until analysis. Total cholesterol, triglycerides and HDL-cholesterol were quantified by standard enzymatic-colorimetric methods [17-19]. LDL-cholesterol was calculated using the Friedewald formula. Lipoprotein (Lp) (a) and homocysteine were measured using commercial assays while the rheumatoid factor by ELISA (IgM isotype, positive $\geq 20$ ). C-reactive protein (CRP) was determined by a highsensitivity nephelometric method [20].

In order to assess the prevalence of each individual component of ALP in both groups of subjects, according the most recent inter- national guidelines [10,21], we considered low HDL-cholesterol levels those $<1.03 \mathrm{mmol} / \mathrm{L}$ (i.e. $<40 \mathrm{mg} / \mathrm{dL}$ ) in men and $<1.29$ (i.e. $<50 \mathrm{mg} / \mathrm{dL}$ ) in women and elevated triglyceride concentrations those $>1.69 \mathrm{mmol} / \mathrm{L}$ (i.e. $>150 \mathrm{mg} / \mathrm{dL}$ ) [21]. Levels of small, dense LDL (e.g. LDL-IIIA + LDL-IIIB, + LDL-IVA + LDL-IVB, \% of LDL stain) were considered to be increased in patients with values greater than mean +2 SD of the values of controls, as already described $[22,23]$. Further, elevated LDL-cholesterol levels were considered those $>4.1 \mathrm{mmol} / \mathrm{L}$ (i.e. $>160 \mathrm{mg} / \mathrm{dL}$ ) while high Lp(a) concentrations were considered those $>30 \mathrm{mg} / \mathrm{dL}$ [10]; elevated triglyceride/HDLcholesterol ratio was considered as $>3.5[24]$.

\subsection{Nondenaturing polyacrylamide gradient gel electrophoresis}

Nondenaturing polyacrylamide gradient gel electrophoresis (GGE) of plasma was performed at University Hospital Zurich, Switzerland in the laboratory of K.B. at $10-14{ }^{\circ} \mathrm{C}$ in $2-6 \%$ polyacrylamide gradient gels. Gels were manufactured by C.B.S. Scientific Company, Solana Beach, CA, USA and subjected to electrophoresis for $24 \mathrm{~h}$ at $125 \mathrm{~V}$ in tris borate buffer ( $\mathrm{pH} 8.3$ ) as described elsewhere [25]. Gels were fixed and stained for lipids in a solution containing oil red $\mathrm{O}$ in $60 \%$ ethanol at $55^{\circ} \mathrm{C}$. Gels were placed on a light source and photographed using a Luminescent Image Analyzer, LAS-3000 of Fujifilm, detection using white transmitted light source. Migration distance for each absorbance peak was determined and the molecular diameter corresponding to each peak was calculated from a calibration curve generated from the migration distance of size standards of known diameter, which includes carboxylated latex beads (Duke Scientific, Palo Alto, CA), thyroglobulin and apoferritin (HMW Std, Pharmacia, Piscataway, NJ) having molecular diameter of $380 \AA$, $170 \AA ̊$ and $122 \AA$, respectively, and lipoprotein calibrators of previously determined particle size. LDL peak particle size was measured as the particle diameter of the predominant peak and LDL subclass distribution (LDL I, IIA, IIB, IIIA, IIIB, IVA and IVB) as percentage of total LDL was calculated as previously described [25].

\subsection{Statistical analysis}

Statistical analyses were performed using Statview ${ }^{\circledR} 5.0$ (SAS Institute Inc., Cary, NC, USA). Univariate analyses were performed using non-parametric Mann-Whitney test for numeric variables, while the differences in the prevalence for nominal variables were analyzed by chi-square test. Correlation analyses were performed using the Spearman rank correlation method.

Table 1

Clinical and laboratory characteristics in all subjects.

\begin{tabular}{|c|c|c|c|}
\hline & Controls $(n=22)$ & $p=$ & Patients $(n=25)$ \\
\hline Age (years) & $46 \pm 4$ & $n s$ & $47 \pm 8$ \\
\hline Female gender $(n)(\%)$ & $18(82)$ & $n s$ & $19(76)$ \\
\hline Body-mass index $\left(\mathrm{kg} / \mathrm{m}^{2}\right)$ & $25 \pm 2$ & $n s$ & $25 \pm 4$ \\
\hline C-reactive protein $(\mathrm{mg} / \mathrm{L})$, median $(\mathrm{Q} 1-\mathrm{Q} 3)$ & $0.35(0.01-0.91)$ & $<0.0001$ & $1.80(1.42-2.79)$ \\
\hline Total cholesterol $(\mathrm{mmol} / \mathrm{L})$ & $4.6 \pm 0.7$ & $n s$ & $4.8 \pm 1.1$ \\
\hline Triglycerides $(\mathrm{mmol} / \mathrm{L})$ & $1.0 \pm 0.5$ & $<.0001$ & $1.8 \pm 0.5$ \\
\hline HDL-cholesterol (mmol/L) & $1.4 \pm 0.2$ & 0.0027 & $1.2 \pm 0.2$ \\
\hline LDL-cholesterol (mmol/L) & $2.7 \pm 0.7$ & ns & $2.8 \pm 0.9$ \\
\hline Triglycerides/HDL-cholesterol ratio & $1.8 \pm 1.0$ & $<0.0001$ & $3.6 \pm 1.2$ \\
\hline Lp(a) (mg/dL), median (Q1-Q3) & $15(11-21)$ & $n s$ & $25(11-102)$ \\
\hline Homocysteine $(\mathrm{mmol} / \mathrm{L})$ & $10 \pm 4$ & $n s$ & $10 \pm 3$ \\
\hline LDL size (angstrom) & $281 \pm 9$ & $<0.0001$ & $264 \pm 7$ \\
\hline Total small, dense LDL (LDL-III and -IV) (\%) & $32 \pm 5$ & $<0.0001$ & $41 \pm 6$ \\
\hline
\end{tabular}

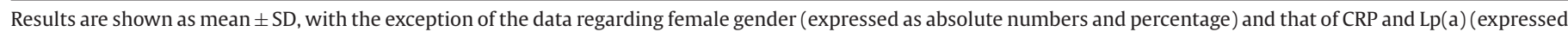
as median with interquartile range).

DAS-28: disease activity for 28 joint indices score [16]. Q: quartile.

Italic values show the statistical data. 


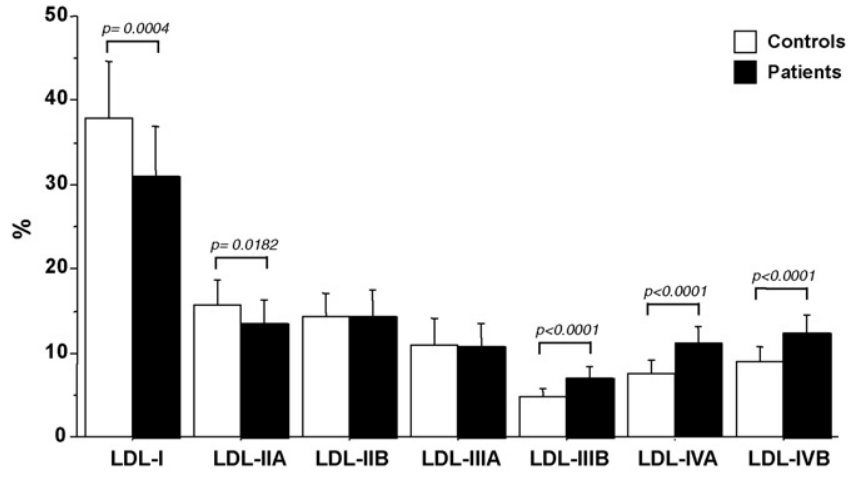

Fig. 1. LDL subclasses in patients with early RA and controls, as assessed by whole plasma nondenaturing polyacrylamide gradient gel electrophoresis. Results are presented as mean $+\mathrm{SD}$.

\section{Results}

All patients with early RA were rheumatoid factor positive (rheumatoid factor: $279 \pm 116 \mathrm{IU} / \mathrm{mL}$ ) and displayed high disease activity scores (DAS-28: $6.2 \pm 1.6$ ) (data not shown). According to the inclusion criteria, patients with early RA and controls were matched for age and BMI (Table 1). Expectedly, patients had higher CRP concentrations than controls (median 1.80 vs. $0.35 \mathrm{mg} / \mathrm{L}, p<0.0001)$, triglyceride levels were also higher $(1.8 \pm 0.5$ vs. $1.0 \pm 0.5 \mathrm{mmol} / \mathrm{L}, p<0.0001)$ and HDL-cholesterol concentrations lower ( $1.2 \pm 0.2$ vs. $1.4 \pm 0.2 \mathrm{mmol} / \mathrm{L}, p=0.0027)$ as was $\mathrm{LDL}$ particle size $(264 \pm 7$ vs. $281 \pm 9 \AA, p<0.0001)$, due to reduced LDL-I ( $31 \pm 6$ vs. $38 \pm 7 \%, p=0.0004)$ and LDL-IIA ( $14 \pm 3$ vs. $16 \pm 3 \%, p=0.0182$ ) and increased LDL-IIIB ( $7 \pm 1$ vs. $5 \pm 1 \%$ ), IVA $(11 \pm 2$ vs. $8 \pm 2 \%)$ and - IVB particles ( $12 \pm 2$ vs. $9 \pm 2 \%$, $(p<0.0001$ for all, see Fig. 1); therefore, levels of small, dense LDL were increased in patients vs. controls ( $41 \pm 6$ vs. $32 \pm 6 \%$, $p<0.0001$ ).

High concentrations of small, dense LDL with normal triglyceride and HDL-cholesterol concentrations were found in two RA patients (8\%). In addition, we have found that the triglyceride/HDLcholesterol ratio was increased in patients $(3.6 \pm 1.2$ vs. $1.8 \pm 1.0$, $p<0.0001)$ and a high ratio was found in 15 patients (68\%) vs. 3 controls $(14 \%)(p=0.0011)$. Yet, the concordance between elevated small, dense LDL and high ratio was low (60\%) and the correlation between the two parameters was not significant in both patients and controls (data not shown). We also calculated the prevalence of different forms of atherogenic dyslipidemia in patients vs. controls (see Table 2), with significant differences in the prevalence of high triglycerides (64 vs. 5\%, $p<0.0001$ ), low HDL-cholesterol (68 vs. $23 \%, p=0.0019$ ), elevated small, dense LDL ( 40 vs. $0 \%, p=0.0005$ ) and high $\operatorname{Lp}(\mathrm{a})$ ( 32 vs. $5 \%, p=0.0112$ ). Eight patients vs. none in the control group $(p=0.0036)$ showed the complete form of ALP, i.e. the
Table 2

Prevalence of different forms of atherogenic dyslipidemia in all subjects.

\begin{tabular}{|c|c|c|c|}
\hline & Controls $(n=22)$ & $p=$ & Patients $(n=25)$ \\
\hline $\begin{array}{l}\text { High LDL-cholesterol (>4.1 mmol/L) } \\
\text { (n) }(\%)\end{array}$ & $1(5)$ & $n s$ & $3(12)$ \\
\hline High triglycerides (n) (\%) & $1(5)$ & $<0.0001$ & $16(64)$ \\
\hline Low HDL-cholesterol (n) (\%) & $5(23)$ & 0.0019 & $17(68)$ \\
\hline $\begin{array}{l}\text { High small, dense LDL particles (n) } \\
(\%)\end{array}$ & $0(0)$ & 0.0005 & $10(40)$ \\
\hline $\begin{array}{l}\text { High triglycerides/HDL-cholesterol } \\
\text { ratio }(\mathrm{n})(\%)\end{array}$ & $3(14)$ & 0.0011 & $15(68)$ \\
\hline Elevated Lp(a) (>30 mg/dL) (n) (\%) & $1(5)$ & 0.0112 & $8(32)$ \\
\hline $\begin{array}{l}\text { Atherogenic lipoprotein phenotype } \\
\text { (n) }(\%)\end{array}$ & $0(0)$ & 0.0036 & $8(32)$ \\
\hline
\end{tabular}

Italic values show the statistical data.

concomitant presence of high triglycerides, low HDL-cholesterol and elevated small, dense LDL.

Spearman correlation analysis was performed to assess potential correlations between LDL size and subclasses and age, BMI and biochemical parameters in all subjects (see Table 3). CRP and triglycerides were inversely correlated with LDL size and LDL-I and positively with LDL-IIIB, -IVA and-IVB; by contrast, HDL-cholesterol was positively correlated with LDL size and LDL-I and inversely with LDL-IIIB, -IVA and-IVB. These correlations remained significant after adjustment for age and BMI (data not shown). No significant correlations were found with age, BMI, total- and LDL-cholesterol, $\mathrm{Lp}(\mathrm{a})$ and homocysteine. We have also performed correlation analysis separately for the groups of patients and controls (data not shown) and found the following significant associations in the group of patients only: LDL size with triglycerides $(r=-.585$, $p=0.0042)$ and LDL size with CRP $(r=-.612, p=0.0032)$.

\section{Discussion}

It has been shown that subjects with early RA may have an atherogenic lipid profile, with increased LDL-cholesterol and LDL:HDLcholesterol ratio [5]. In the present study we have extended such preliminary observation investigating in this category of patients LDL size and all seven LDL subclasses, as well as the full ALP. In fact, LDL are very heterogeneous particles and comprise multiple distinct subclasses that differ in size, density, physicochemical composition, metabolic behaviour and atherogenicity, with at least four major subspecies: large LDL-I, medium LDL-II, small LDL-III and very small LDL-IV [26]. The predominance of small, dense LDL has been associated with up to a seven fold increased risk for coronary artery disease [7] and several reasons have been suggested for the atherogenicity of small dense LDL. In relation to larger, more buoyant LDL, small dense LDL are taken up more easily by arterial tissue, have decreased sialic acid content and receptor-mediated uptake, as well as increased oxidative susceptibility and reduced antioxidant concentrations [9]. Therefore, screening for the presence of

Table 3

(Online Supplementary Material Only). Spearman correlations between LDL size and subclasses and age, BMI and biochemical parameters in all subjects.

\begin{tabular}{|c|c|c|c|c|c|c|c|c|c|}
\hline & Age & BMI & C-reactive protein & Total cholesterol & Triglycerides & HDL-cholesterol & LDL-cholesterol & Lp (a) & Homocysteine \\
\hline LDL size & -.008 & -.151 & $-.645^{a}$ & -.102 & $-.664^{a}$ & $.457^{\dagger}$ & -.012 & -.234 & .014 \\
\hline LDL-I & .079 & -.241 & $-.429^{\dagger}$ & .161 & $-.396^{*}$ & $.450^{\dagger}$ & .181 & -.104 & -.063 \\
\hline LDL-IIA & .007 & -.030 & .012 & .156 & -.154 & .221 & .162 & -.067 & -.198 \\
\hline LDL-IIB & -.185 & .130 & .241 & .071 & .107 & -.139 & .087 & .092 & -.123 \\
\hline LDL-IIIA & -.245 & .036 & .011 & -.206 & .141 & -.148 & -.238 & -.056 & .073 \\
\hline LDL-IIIB & .078 & .169 & $.380^{*}$ & -.217 & $.381^{*}$ & $-.437^{\dagger}$ & -.243 & .170 & .193 \\
\hline LDL-IVA & .110 & .191 & $.377^{*}$ & -.205 & $.399^{*}$ & $-.481^{\dagger}$ & -.222 & .154 & .173 \\
\hline LDL-IVB & .092 & .227 & $.316^{*}$ & -.156 & $.383^{*}$ & -.456 & -.168 & .075 & .182 \\
\hline
\end{tabular}

In bold the relationships that reached the statistical significance.

a $<0.0005$.

$p<0.05$.

$<0.005$. 
small, dense LDL may potentially identify subjects with higher vascular risk and may contribute in directing specific interventions of cardiovascular prevention

Patients with RA have a higher cardiovascular risk than the general population and lipid and lipoproteins alterations are common and usually include decreased HDL-cholesterol and increased LDL:HDLcholesterol ratio [4,5]. In the present study patients with early RA showed increased triglyceride concentrations and reduced HDL-cholesterol levels than controls, while total- and LDL-cholesterol did not differ between the two groups. Elevated triglycerides and reduced HDL-cholesterol represents two lipid abnormalities usually accompanied by increased levels of small, dense LDL in the ALP. We found that patients with early RA had lower LDL size than controls and LDL subclass analysis revealed that this was due to changes in distinct LDL subspecies, with reduced LDL-I and -IIA subclasses and increased LDL-IIIB, -IVA and -IVB particles. Interestingly, $40 \%$ of patients with early RA showed elevated levels of small, dense LDL, though we recognize the limitation of analyzing \% data, which is that they must sum to 100 , so technically there can be at most only n-1 different fractions.

These findings are somewhat consistent with what previously reported by Hurt-Camejo et al. [11]. These authors interestingly found that patients with long-term RA showed lower LDL size due to increased levels of small, dense LDL particles; yet, in this study, about $90 \%$ of patients received therapies for the disease and this potentially limits the utility of the information we have on atherogenic dyslipidemia in RA. In fact, although drug treatment in RA is in general beneficial on plasma lipids, it seems that long-term anti-tumor necrosis factor-alpha therapies may induce some pro-atherogenic changes in lipid profile [12-14]. Since the triglycerides/HDL ratio has been proposed as a simple way to identify subjects with a predominance of small, dense LDL [24,27], we further assessed the relationships between these two lipid parameters. The concordance between elevated small, dense LDL and high triglycerides/HDL ratio was only $60 \%$ and this was confirmed by the non-significant correlation between the two parameters, supporting the concept that lipid ratios cannot represent fully reliable markers of lipoprotein abnormalities [10].

We have found by correlation analysis that such alterations in LDL subclasses were strongly associated with the extent of inflammation, as indirectly measured by CRP levels, in all subjects; further, in the group of RA patients, despite the small cohort, there was a significant inverse correlation between LDL size and CRP concentrations. Interestingly, significant associations between markers of inflammation and LDL subclasses have been reported in another rheumatic disease, i.e. the systemic lupus erythematosus [28]. Besides correlation analysis, we directly investigated the prevalence of complete and incomplete forms of ALP in patients and controls; we found that elevated triglycerides, low HDL-cholesterol and high small, dense LDL are very common in patients with early RA and the complete form of ALP was found in 1/3 of patients. Overall, our findings suggest that more than the traditional lipids should be potentially taken into account in patients with early RA for a better management of their disease. Since the therapeutic modulation of ALP and particularly of distinct LDL subspecies (such as small, dense LDL particles) is of great benefit in reducing cardiovascular risk [8,29-30], their measurement should be potentially extended to this category of patients.

In fact, increasing evidence suggest that treatment of atherogenic dyslipidemia should be considered as a part of cardiovascular risk management in RA [31]; in addition, recent studies have shown the beneficial effects of statins in such patients, particularly on disease activity, swollen joint count and endothelial dysfunction (as reviewed in [32]). Since statin use in RA is now suggested, it should be highlighted that statins have shown differential effects on the "quantity" and the "quality" of LDL $[30,33]$ and, therefore, the analysis of a more detailed lipid profile may contribute to assess a personalized therapy, choosing the best statin to be used.

Yet, it is still questioned whether atherogenic dyslipidemia precedes or follows onset of RA [34]. Van Halm et al. [35] have interestingly determined plasma lipids and lipoproteins in blood donors who later developed RA vs. age- and gender-matched controls: they found that future RA patients had higher levels of total-cholesterol, triglycerides and apoproteinB as well as reduced HDL-cholesterol concentrations at least ten years before the onset of symptoms. Since other authors have shown an active modulating role of lipids in inflammation [36,37], on the basis of available evidence it cannot be excluded that atherogenic dyslipidemia may precede the onset of RA.

A potential limitation of the present study is the relative small cohorts of patients and controls. Yet, it should be noted that despite the small numbers, the observation appears to be solid, as we found highly significant differences in several parameters, including LDL size and small, dense LDL subclasses. Another potential limitation of the present study is represented by the lack of analysis of apolipoproteins (such as A1 and B) or phospholipase A2; yet, we reasoned not to assess such parameters as they have already been analyzed in patients with early RA [5,38]. Finally, although we have assessed (by correlation analysis) the associations between LDL size and subclasses with clinical and biochemical parameters, we did not assess independent predictors of small, dense LDL (by multivariate analyses): such analyses were beyond the specific aim of the present study and the number of patients had to be extended considerably.

In conclusion, we found in the present study that drug-naïve patients with early RA (i.e. who had a disease durations $<1$ year and not prior treatment for it), in relation to age- and BMI-matched controls showed reduced LDL size due to changes in their LDL subclass distribution, with a strong reduction in larger particles and a concomitant increase in the smallest, most dense LDL. Further, we found that $40 \%$ of patients with early RA showed elevated levels of small, dense LDL and about 1/3 the complete form of ALP. Yet, whether these findings affect the atherogenic process and the clinical endpoints in this category of subjects remains to be determined by future prospective studies.

\section{Acknowledgements}

We wish to thank Cornelia Zwimpfer for laboratory skills in performing gradient gel electrophoresis. M. Rizzo and G.B. Rini were recipients of the "ex-60\%" grant from University of Palermo, Italy.

\section{Appendix A. Supplementary data}

Supplementary data associated with this article can be found, in the online version, at doi:10.1016/j.atherosclerosis.2009.07.015.

\section{References}

[1] Wallberg-Jonsson S, Ohman ML, Dahlqvist SR. Cardiovascular morbidity and mortality in patients with seropositive rheumatoid arthritis in Northern Sweden. J Rheumatol 1997;24:445-51.

[2] Del Rincon I, Williams K, Stern MP, Freeman GL, Escalante A. High incidence of cardiovascular events in a rheumatoid arthritis cohort not explained by traditional cardiac risk factors. Arthritis Rheum 2001;44:2737-45.

[3] Boers M, Dijkmans B, Gabriel S, Maradit-Kremers H, O’Dell J, Pincus T. Making an impact on mortality in rheumatoid arthritis: targeting cardiovascular comorbidity. Arthritis Rheum 2004;50:1734-9.

[4] Van Doornum S, McColl G, Wicks IP. Accelerated atherosclerosis: an extraarticular feature of rheumatoid arthritis? Arthritis Rheum 2002;46:862-73.

[5] Georgiadis AN, Papavasiliou EC, Lourida ES, et al. Atherogenic lipid profile is a feature of patients with early rheumatoid arthritis: effect of early treatment-a prospective, controlled study. Arthritis Res Ther 2006;8:R82.

[6] Austin MA, King MC, Vranizan KM, Krauss RM. Atherogenic lipoprotein phenotype. A proposed genetic marker for coronary heart disease risk. Circulation 1990;82:495-506. 
[7] Superko HR. Beyond LDL cholesterol reduction. Circulation 1996:94:2351-4.

[8] Nesto RW. Beyond low-density lipoprotein: addressing the atherogenic lipid triad in type 2 diabetes mellitus and the metabolic syndrome. Am J Cardiovasc Drugs 2005;5:379-87.

[9] Rizzo M, Berneis K. Low-density-lipoproteins size and cardiovascular risk assessment. QJM-Int J Med 2006;99:1-14.

[10] National Cholesterol Education Program (NCEP). Expert Panel on Detection, Evaluation, and Treatment of High Blood Cholesterol in Adults (Adult Treatment Panel III). Third Report of the National Cholesterol Education Program (NCEP) Expert Panel on Detection, Evaluation, and Treatment of High Blood Cholesterol in Adults (Adult Treatment Panel III) final report. Circulation 2002; 106:3143-421. 23.

[11] Hurt-Camejo E, Paredes S, Masana L, et al. Elevated levels of small, low-density lipoprotein with high affinity for arterial matrix components in patients with rheumatoid arthritis: possible contribution of phospholipase A2 to this atherogenic profile. Arthritis Rheum 2001;44:2761-7.

[12] Irace C, Mancuso G, Fiaschi E, Madia A, Sesti G, Gnasso A. Effect of anti TNFalpha therapy on arterial diameter and wall shear stress and HDL cholesterol. Atherosclerosis 2004;177:113-8.

[13] Allanore Y, Kahan A, Sellam J, Ekindjian OG, Borderie D. Effects of repeated infliximab therapy on serum lipid profile in patients with refractory rheumatoid arthritis B. Clin Chim Acta 2006;365:143-8.

[14] Rantapaa-Dahlqvist S, Engstrand S, Berglin E, Jonson O. Conversion towards an atherogenic lipid profile in rheumatoid artritis patients during long-term infliximab therapy. Scand J Rheumatol 2006;35:107-11.

[15] Arnett FC, Edworthy SM, Bloch DA, et al. The American Rheumatism Association 1987 revised criteria for the classification of rheumatoid arthritis. Arthritis Rheum 1988;31:315-24.

[16] Prevoo ML, van't Hof MA, Kuper HH, van Leeuwen MA, van de Putte LB, van Riel PL. Modified disease activity scores that include twenty-eight-joint counts. Development and validation in a prospective longitudinal study of patients with rheumatoid arthritis. Arthritis Rheum 1995;38:44-8.

[17] Allain CC, Poon LS, Chan CS, Richmond W, Fu PC. Enzymatic determination of total serum cholesterol. Clin Chem 1974;20:470-5.

[18] Nagele U, Hagele EO, Sauer G, et al. Reagent for the enzymatic determination of serum total triglycerides with improved lipolytic efficiency. J Clin Chem Clin Biochem 1984;22:165-74.

[19] Warnick GR, Nguyen T, Albers JJ. Comparison of improved precipitation methods for quantification of high density lipoprotein cholesterol. Clin Chem $1985 ; 31: 217-22$

[20] Montagne P, Laroche P, Cuilliere ML, Varcin P, Duheille J. Microparticleenhanced nephelometric immunoassay for human C- reactive protein. J Clin Lab Anal 1992;1:24-9.

[21] Grundy SM, Cleeman JI, Daniels SR, et al. American Heart Association; National Heart, Lung, and Blood Institute. Diagnosis and management of the metabolic syndrome: an American Heart Association/National Heart, Lung, and Blood Institute Scientific Statement. Circulation 2005;112:2735-52, 32.
[22] Rizzo M, Pernice V, Frasheri A, Berneis K. Atherogenic lipoprotein phenotype and LDL size and subclasses in patients with peripheral arterial disease. Atherosclerosis 2008;197:237-41.

[23] Rizzo M, Berneis K, Altinova AE, et al. Atherogenic lipoprotein phenotype and LDL size and subclasses in women with gestational diabetes. Diab Med 2008;25:1406-11.

[24] McLaughlin T, Reaven G, Abbasi F, et al. Is there a simple way to identify insulinresistant individuals at increased risk of cardiovascular disease? Am J Cardiol 2005;96:399-404

[25] Krauss R, Burke D. Identification of multiple subclasses of plasma low density lipoproteins in normal humans. J Lipid Res 1982;23:97-104.

[26] Berneis KK, Krauss RM. Metabolic origins and clinical significance of LDL heterogeneity. J Lipid Res 2002;43:1363-79.

[27] Dobiásová M, Frohlich J. The plasma parameter log (TG/HDL-C) as an atherogenic index: correlation with lipoprotein particle size and esterification rate in apoB-lipoprotein-depleted plasma (FER(HDL)). Clin Biochem 2001;34 583-8.

[28] Chung CP, Oeser A, Raggi P, et al. Lipoprotein subclasses and particle size determined by nuclear magnetic resonance spectroscopy in systemic lupus erythematosus. Clin Rheumatol 2008;27:1227-33.

[29] Barter P. Options for therapeutic intervention: how effective are the different agents? Eur Heart J Suppl 2006;8:F47-53.

[30] Gazi IF, Tsimihodimos V, Tselepis AD, Elisaf M, Mikhailidis DP. Clinical importance and therapeutic modulation of small dense low-density lipoprotein particles. Expert Opin Biol Ther 2007;7:53-72.

[31] Nurmohamed MT. Atherogenic lipid profiles and its management in patients with rheumatoid arthritis. Vasc Health Risk Manag 2007;3:845-52.

[32] Paraskevas KI. Statin treatment for rheumatoid arthritis: a promising novel indication. Clin Rheumatol 2008;27:281-7.

[33] Rizzo M, Berneis K. The clinical relevance of low-density-lipoproteins size modulation by statins. Cardiovasc Drugs Ther 2006;20:205-17.

[34] Nurmohamed MT, Dijkmans BA. Dyslipidaemia, statins and rheumatoid arthritis. Ann Rheum Dis 2009;68:453-5.

[35] van Halm VP, Nielen MM, Nurmohamed MT, et al. Lipids and inflammation serial measurements of the lipid profile of blood donors who later developed rheumatoid arthritis. Ann Rheum Dis 2007;66:184-8.

[36] Hyka N, Dayer JM, Modoux C, et al. Apolipoprotein A-I inhibits the production of interleukin-1beta and tumor necrosis factor-alpha by blocking contactmediated activation of monocytes by T lymphocytes. Blood 2001;97:2381-9.

[37] Ashby DT, Rye KA, Clay MA, Vadas MA, Gamble JR, Barter PJ. Factors influencing the ability of HDL to inhibit expression of vascular cell adhesion molecule-1 in endothelial cells. Arterioscler Thromb Vasc Biol 1998;18:1450-5.

[38] Lourida ES, Georgiadis AN, Papavasiliou EC, Papathanasiou AI, Drosos AA, Tselepis AD. Patients with early rheumatoid arthritis exhibit elevated autoantibody titers against mildly oxidized low-density lipoprotein and exhibit decreased activity of the lipoprotein-associated phospholipase A2. Arthritis Res Ther 2007;9:R19. 